\title{
Freezing of gait is associated with cortical thinning in mesial frontal cortex
}

\author{
Miroslav Vastika, Pavel Hok ${ }^{\mathrm{a}}$, Jan Valosek ${ }^{\mathrm{a}, \mathrm{b}}$, Petr Hlustika, Katerina Mensikova ${ }^{\mathrm{a}}$, Petr Kanovsky ${ }^{\mathrm{a}}$
}

\begin{abstract}
Aims. The relationship between freezing of gait (FOG) and regional brain atrophy has been intensively investigated, but it is still not clearly understood. The study objective was to test whether grey matter (GM) atrophy contributes to FOG in Parkinson's disease (PD) using a surface-based algorithm.

Methods. We investigated 21 patients with PD, 11 with FOG and 10 without FOG. Both groups were assessed using a FOG questionnaire and Hoehn and Yahr staging. High resolution T1-weighted brain images were acquired for each subject using a 1.5T MRI scanner. A surface-based method implemented in FreeSurfer was used to quantify the GM atrophy. A vertex-wise and region of interest (ROI) comparison of spatially normalized subject data using a general linear model and the Wilcoxon rank sum test were to assess significant group differences.

Results. Higher global levels of cortical atrophy were detected in freezers, although this was not statistically significant. The vertex-wise analysis revealed significant local reduction in grey matter thickness in the left supplementary motor area, middle/anterior cingulate cortex, temporal pole and right frontal operculum in freezers at $P<0.001$, uncorrected. The ROI analysis of average thickness confirmed the regional atrophy in bilateral anterior and posterior cingulate cortices. No significant relative regional cortical atrophy was observed in non-freezers.

Conclusion. FOG was associated with regional cortical atrophy, especially in mesial frontal and cingulate cortices. Our findings provide additional evidence that the development of FOG in patients with PD is associated with local structural cortical changes.
\end{abstract}

Key words: Parkinson's disease, freezing of gait, brain atrophy, anterior cingulate cortex, supplementary motor area

Received: May 5, 2017; Accepted with revision: July 26, 2017; Available online: September 13, 2017

https://doi.org/10.5507/bp.2017.035

${ }^{a}$ Department of Neurology, Faculty of Medicine and Dentistry, Palacky University Olomouc and University Hospital Olomouc, Czech Republic ${ }^{b}$ Department of Biomedical Engineering, University Hospital Olomouc, Czech Republic

Corresponding author: Miroslav Vastik, e-mail:vastik1@seznam.cz

\section{INTRODUCTION}

FOG is an episodic inability to produce effective steps in the absence of other causes besides parkinsonism or high-level gait disorders ${ }^{1}$. FOG is a characteristic feature of PD but it does not occur universally ${ }^{2}$. It occurs more in the later stages, generally in about 40 to $60 \%$ of patients with Parkinson's disease. It is more common (up to $90 \%$ ) when the effect of dopaminergic medication decreases. The episodes of FOG are mitigated by levodopa therapy. Improvement after the use of medication, so-called "off freezing", but it may be present also in the state of good compensation - "on freezing". Patients with freezing have increased variability in walking (stride-to-stride variability markedly increases in PD subjects with FOG compared to those without FOG) even outside freezing episodes. PD subjects with FOG have a continuous gait disturbance: the ability to regulate the stride-to-stride variations in gait timing and maintain a stable walking rhythm is markedly impaired in subjects with freezing of gait $^{3}$. The exact pathophysiological mechanism of FOG is not known. The responsible pathophysiological substrate of FOG is likely complex.

Disturbances in cortical functions leading to FOG may be associated with focal brain atrophy, which can be measured using voxel-based morphometry (VBM). This method compares volume of the grey matter ob- tained from whole-brain segmentation of high resolution anatomical magnetic resonance imaging (MRI) data. Although the methods for group comparison of VBM data are highly sophisticated, they are prone to bias from various optional user settings, leading potentially to inconsistencies ${ }^{4}$. Several previous studies investigated the brain alterations associated with FOG in patients with PD using VBM, but they yielded variable outcomes ${ }^{5-11}$. Because of these differences, potentially related to VBM methodology, it may be worth exploring cortical atrophy with a different method.

In contrast to VBM measures, surface-based approach provides a sensitive tool for investigation of cortical atrophy by breaking down measures of local cortical volume into separate and almost orthogonal components of thickness and surface area ${ }^{12,13}$. The method implemented in the open source software FreeSurfer can be used to extract the cortical envelope from a T1-weighted MRI scan and to register the individual cortex surfaces to surface-based anatomical atlases ${ }^{14,15}$ allowing for surface-based groupwise comparisons. Here, we employed FreeSurfer ${ }^{16}$ to compare the regional cortical thickness in PD patients with and without FOG using high-resolution anatomical T1-weighted imaging. 


\section{MATERIALS AND METHODS}

The study was approved by the Ethics Committee of the University Hospital Olomouc and the Faculty Medicine Palacky University in Olomouc. All patients gave their written informed consent before any study procedure.

We investigated 21 patients with PD, 11 with FOG $(\mathrm{FOG}+)$ and 10 without FOG (FOG-). Patients were 6080 years old with disease duration of 5-15 years. The average age of patients with and without FOG was 71.4 and 70.2 respectively. The groups did not differ significantly with respect to age (Mann-Whiney U-test, $P=0.673$ ). The inclusion criteria were: 1) subjects with idiopathic PD, as defined by the UK Brain Bank criteria ${ }^{17} 2$ ) a Hoehn and Yahr Stage of 4 or better when "on"; 3) stable PD medication (patients on levodopa treatment) for at least 30 days; 4) FOG questionnaire (FOGQ) score more than 4 for FOG+ group and FOGQ score 0 for FOG- group. Subjects were excluded if their disease was recently diagnosed (disease duration less than 2 years), if they had brain surgery in the past, or if they had clinically significant comorbidities likely to affect gait including diabetes, rheumatic or orthopaedic disease, dementia, depression, or history of stroke, as determined by clinical and radiological evaluation.

To compare and contrast subjects with and without freezing, the study population was characterized with respect to age, gender, duration of symptoms, MiniMental State Exam (MMSE) scores ${ }^{18}$ and the Unified Parkinson's disease Rating Scale (UPDRS) scores (part I-III) (ref. ${ }^{19}$ ) assessed during standard neurological examination in the morning at least $12 \mathrm{~h}$ after they took their last anti-parkinsonian medication. Subscore indices of motor function were also determined from the UPDRS $\left(\right.$ ref. $\left.{ }^{20}\right)$. The scores in the UPDRS part II and part III were compared also after the removal of the questions 2.13 and 3.11 (both questions are specific for freezing of gait) to assess group differences unrelated to freezing. FOG was evaluated using FOG questionnaire, which is able to quantify self-report history of mobility, gait disturbances, and freezing ${ }^{21}$. The new freezing of gait questionnaire was published in 2009 by Nieuwboer et al. ${ }^{22}$.

The quantitative data are described using descriptive statistics (median, minimum, maximum, mean, and standard deviation - SD). Comparison of groups for quantitative and ordinal variables (Hoehen and Yahr) was made using the non-parametric Mann-Whitney U-test for skewed distributions. For normally distributed variables, the $t$ test was used. Qualitative data were described using absolute and relative frequencies (percent), and the differences were tested using Fisher's exact test.

\section{Data acquisition}

Magnetic resonance imaging data were acquired using available 1.5-Tesla scanners (Avanto and Symphony, Siemens, Erlangen, Germany) with standard head coils. Patients were scanned in supine position, they were asked not to move and their head was immobilized with cush- ions to ensure maximum comfort and to minimize head movement. The MRI protocol included fluid-attenuated inversion recovery (FLAIR) images to visualize brain lesions and a high-resolution 3D anatomical scan using Magnetization Prepared Rapid Acquisition Gradient Echo sequence (MPRAGE) with optimal parameters for each scanner, i.e., for Symphony: repetition time/echo time (TR/TE) 1900/3.93 ms, inversion time (TI) ms 1100 ms, flip angle (FA) $15^{\circ}$, sagittal slice orientation, 144 slices, slice thickness $1.20 \mathrm{~mm}$, field of view (FOV) 211 $\mathrm{mm} \times 211 \mathrm{~mm}$, matrix $192 \times 192,8 \%$ oversampling in the phase-encoding direction, fat saturation ON; for Avanto: repetition time/echo time (TR/TE) 2130/5.53 ms, inversion time (TI) ms $1100 \mathrm{~ms}$, flip angle (FA) $15^{\circ}$, sagittal slice orientation, 144 slices, slice thickness $1.25 \mathrm{~mm}$, field of view (FOV) $250 \mathrm{~mm} \times 250 \mathrm{~mm}$, matrix $256 \times 256,75 \%$ sampling the phase-encoding direction, fat saturation $\mathrm{ON}$.

Each subject was scanned twice within 4 weeks. The images were visually checked for artefacts by an experienced radiologist, and one dataset with better image quality out of the two available was always chosen for the subsequent analysis. One subject in the FOG- group had to be excluded due to severe motion and susceptibility artefacts affecting both scans. The final MR images were acquired at either of the available scanners, however the number of eligible examinations at each scanner was roughly similar in both groups, i.e., 7 v. 2 in FOG-, and 7 v. 4 in FOG+, for Avanto and Symphony, respectively.

\section{Analysis}

The preprocessing of T1-weighted images and surface reconstruction was carried out using FreeSurfer ${ }^{12,13}$, version 5.3.0. A standard automated pipeline (recon-all) was used, including removal of non-brain tissue ${ }^{23}$, grey and white matter segmentation with reconstruction of subcortical brain structures ${ }^{24}$, intensity normalization, white matter and pial surface reconstruction using tessellation of the grey/white matter boundary with automated topology correction ${ }^{25}$, followed by surface deformation to optimally place the borders at the location with the greatest shift in intensity ${ }^{12}$. The cortical surface models were then inflated ${ }^{13}$, registered to a spherical atlas based on individual cortical folding patterns ${ }^{26}$, and parcellated into units with respect to gyral and sulcal structure ${ }^{27}$. At each of the following steps, the resulting data were visually checked and manually edited where needed: brain mask estimation, white matter segmentation and pial surface reconstruction.

Vertex-wise and region of interest (ROI) statistical analyses were performed to assess significant group differences. The vertex-wise analysis was carried out using general linear model (GLM) implemented in Qdec, part of FreeSurfer. An unpaired group contrast using a t-test yielded cortical statistical maps thresholded vertex-wise at $P<0.001$ (uncorrected). The ROI analysis was carried out using average values extracted from vertices falling within cortical labels of the cortical parcellation atlas ${ }^{15}$. The thickness values were compared using Mann-Whiney U-test. To exclude the impact of global brain volume, the 
total intracranial volume was compared between groups using Mann-Whiney U-test as well.

\section{RESULTS}

\section{Clinical findings}

Of the 20 patients with PD, 11 were classified as $\mathrm{FOG}^{+}$(patients with freezing of gait/group A) and 10 patients as FOG- (patients without freezing of gait/ group B).

Clinical characteristics are summarized in Table 1. Mann-Whitney U-test showed that Hoehen and Yahr staging was significantly higher $(P=0.045)$ in group FOG + . Mann-Whitney U-test also showed significantly higher score in the UPDRS part II $(P=0.001)$ and III $(P=0.028)$ in group $\mathrm{FOG}^{+}$. The group difference in the UPDRS III was no longer significant after the removal of question $3.11(P=0.084)$, whereas the group difference in the UPDRS II remained significant even after the removal of the question $2.13(P=0.001)$. These results indicate that after the removal of the questions that are specific to FOG, patients with and without FOG achieved comparable scores in the motor examination. The persisting difference in the UPDRS II may be explained by the effect of FOG on motor aspects of daily living, such as hobbies, dressing (for example turning in front of the mirror), and hygiene (for example when the patient is going to the shower).

\section{Imaging}

Higher global levels of cortical atrophy were detected in $\mathrm{FOG}^{+}$, although this was not statistically significant (Table 3). A map of differences in average cortical thickness revealed a predominantly fronto-temporo-parietal pattern of cortical atrophy in $\mathrm{FOG}^{+}$patients (blue overlay in Fig. 1). Bilateral occipital, right dorsal parietal, bilateral middle temporal and orbitofrontal cortices were relatively spared or even more atrophic in the FOG- group (red overlay in Fig. 1).

The statistical vertex-wise comparison detected several foci of significant cortical atrophy in $\mathrm{FOG}^{+}$: left supplementary motor area (Brodmann area - BA 6), middle/ anterior cingulate cortex, temporal pole (BA 38) and frontal operculum $(P<0.001$, uncorrected; blue in Fig. 2, Table 4). No significant vertices with greater atrophy in FOG- were detected.

The ROI analysis of average thickness within cortical

Table 1. Clinical group characteristics - numeric variables.

\begin{tabular}{|c|c|c|c|c|c|c|c|c|c|c|c|}
\hline & \multicolumn{10}{|c|}{ Group } & \multirow{3}{*}{$P^{\mathrm{a}}$} \\
\hline & \multicolumn{5}{|c|}{$\mathrm{FOG}+(\mathrm{n}=11)$} & \multicolumn{5}{|c|}{ FOG- $(n=10)$} & \\
\hline & Median & Min & Max & Mean & SD & Median & Min & Max & Mean & SD & \\
\hline Age & 71.80 & 61.67 & 80.59 & 71.30 & 5.64 & 71.18 & 44.23 & 78.92 & 68.74 & 9.98 & 0.673 \\
\hline FOGQ & 16.00 & 6.00 & 21.00 & 15.91 & 4.46 & & & & & & \\
\hline UPDRS part I & 0.00 & 0.00 & 0.00 & 0.00 & 0.00 & 0.00 & 0.00 & 0.00 & 0.00 & 0.00 & 1.000 \\
\hline UPDRS part II & 20.00 & 10.00 & 30.00 & 19.45 & 5.77 & 8.00 & 6.00 & 15.00 & 9.30 & 3.40 & 0.001 \\
\hline UPDRS part II without 2.13 & 18.00 & 9.00 & 28.00 & 17.60 & 5.60 & 8.00 & 6.00 & 15.00 & 9.30 & 3.40 & 0.001 \\
\hline UPDRS part III & 29.00 & 13.00 & 44.00 & 29.82 & 8.10 & 18.00 & 13.00 & 31.00 & 20.60 & 7.15 & 0.028 \\
\hline UPDRS part III without 3.11 & 27.00 & 12.00 & 42.00 & 27.50 & 7.60 & 18.00 & 13.00 & 31.00 & 20.60 & 7.15 & 0.084 \\
\hline MMSE & 29.00 & 27.00 & 30.00 & 28.55 & 1.21 & 29.50 & 28.00 & 30.00 & 29.30 & 0.82 & 0.141 \\
\hline Disease duration (yrs) & 10.00 & 4.00 & 13.00 & 9.91 & 2.51 & 10.50 & 5.00 & 14.00 & 10.70 & 2.58 & 0.352 \\
\hline FOG duration (yrs) & 5.00 & 2.00 & 7.00 & 4.55 & 1.37 & & & & & & \\
\hline
\end{tabular}

Table shows basic clinical parameters using descriptive statistics and the significance of two-sample statistical comparison.

FOG - freezing of gait, FOG+ - patients with freezing of gait, FOG- - patients without freezing of gait, SD - standard deviation, FOGQ - freezing of gait questionnaire, UPDRS - The Unified Parkinson's Disease Rating Scale, MMSE - Mini Mental State Exam Notes: ${ }^{\mathrm{a}}$ Mann-Whiney U-test

Table 2. Clinical group characteristics - ordinal and categorical variables.

\begin{tabular}{|c|c|c|c|c|c|c|}
\hline & \multicolumn{6}{|c|}{ Group } \\
\hline & & \multicolumn{2}{|c|}{$\mathrm{FOG}^{+}(\mathrm{n}=11)$} & \multicolumn{2}{|c|}{ FOG- $(n=10)$} & \multirow[t]{2}{*}{$P$} \\
\hline & & Count & $\%$ & Count & $\%$ & \\
\hline \multirow[t]{4}{*}{ Hoehen and Yahr } & II & 0 & $0.0 \%$ & 1 & $10.0 \%$ & $0.045^{\mathrm{a}}$ \\
\hline & II. 5 & 0 & $0.0 \%$ & 4 & $40.0 \%$ & \\
\hline & III & 7 & $63.6 \%$ & 3 & $30.0 \%$ & \\
\hline & IV & 4 & $36.4 \%$ & 2 & $20.0 \%$ & \\
\hline \multirow[t]{2}{*}{ Sex } & $\mathrm{F}$ & 2 & $18.2 \%$ & 6 & $60.0 \%$ & $0.080^{\mathrm{b}}$ \\
\hline & M & 9 & $81.8 \%$ & 4 & $40.0 \%$ & \\
\hline
\end{tabular}

Table shows basic clinical parameters using descriptive statistics and the significance of two-sample statistical comparison.

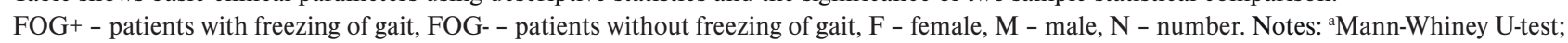
${ }^{\mathrm{b}}$ Fisher's exact test 
Table 3. Differences in global and regional cortical thickness.

\begin{tabular}{lccc}
\hline Region & Median cortical thickness & Median cortical thickness & Wilcoxon rank sum \\
& FOG $(\mathrm{mm} ;<$ range $>)$ & FOG- $(\mathrm{mm} ;<$ range $>)$ & test $(P)$ \\
\hline Cerebrum & $1.962 ;<1.837,2.272>$ & $2.225 ;<1.583,2.338>$ & 0.095 \\
Left Mid-Anterior Cingulate Gyrus and Sulcus & $2.321 ;<1.853,2.745>$ & $2.707 ;<2.166,2.927>$ & $\mathbf{0 . 0 1 6}$ \\
Left Mid-Posterior Cingulate Gyrus and Sulcus & $2.174 ;<1.723,2.428>$ & $2.486<1.745,2.690>$ & $\mathbf{0 . 0 2 5}$ \\
Right Anterior Cingulate Gyrus and Sulcus & $2.237 ;<1.978,2.610>$ & $2.534 ;<2.028,2.684>$ & $\mathbf{0 . 0 4 6}$ \\
Right Mid-Posterior Gyrus and Sulcus & $2.265 ;<1.571,2.723>$ & $2.449 ;<1.865,2.704>$ & $\mathbf{0 . 0 3 8}$ \\
Right Dorsal Posterior Cingulate Gyrus & $2.269 ;<2.000,2.788>$ & $2.564 ;<1.618,2.934>$ & $\mathbf{0 . 0 3 8}$ \\
\hline
\end{tabular}

Table lists global differences in cortical thickness along with significant differences in the region of interest (ROI) analysis in cortical labels according to automated cortical parcellation.

FOG - freezing of gait

Table 4. Local maxima of vertex-wise differences in cortical thickness.

\begin{tabular}{lcc}
\hline Region (BA) & $\begin{array}{c}\text { Coordinates of maximum in the } \\
\text { Talairach space (x, y, z [mm]) }\end{array}$ & $\begin{array}{c}\text { Z value } \\
\text { (FOG+ > FOG-) }\end{array}$ \\
\hline $\begin{array}{l}\text { Left Mid-Anterior Cingulate Gyrus and Sulcus / Superior } \\
\begin{array}{l}\text { Frontal Gyrus (BA 6) } \\
\text { Right Anterior Segment of Lateral Fissure, Horizontal } \\
\text { Ramus / Inferior Frontal Gyrus, Pars triangularis (BA: N/A) }\end{array}\end{array}$ & $-9.76,2.14,46.23$ & -4.30 \\
$\begin{array}{l}\text { Left Circular Sulcus of Insula / Superior Temporal Gyrus } \\
\text { (BA 38) }\end{array}$ & $-45.83,30.50,-1.56$ & -4.16 \\
$\begin{array}{l}\text { Left Anterior Cingulate Gyrus and Sulcus / Superior } \\
\text { Frontal Gyrus (BA 24) }\end{array}$ & $-13.04,28.81,20.44$ & -3.39 \\
\hline
\end{tabular}

Table lists local maxima of significant vertex-wise differences in cortical thickness. The table provides maximum $\mathrm{Z}$ score (normalized $\mathrm{t}$ value of a two-sample t-test), coordinates of the local maximum in the Talairach space and parcellation labels using the cortical atlas ${ }^{14,15}$, respectively, followed by Brodmann area (BA) label ${ }^{28}$ in parenthesis.

BA - Brodmann area; FOG - freezing of gait

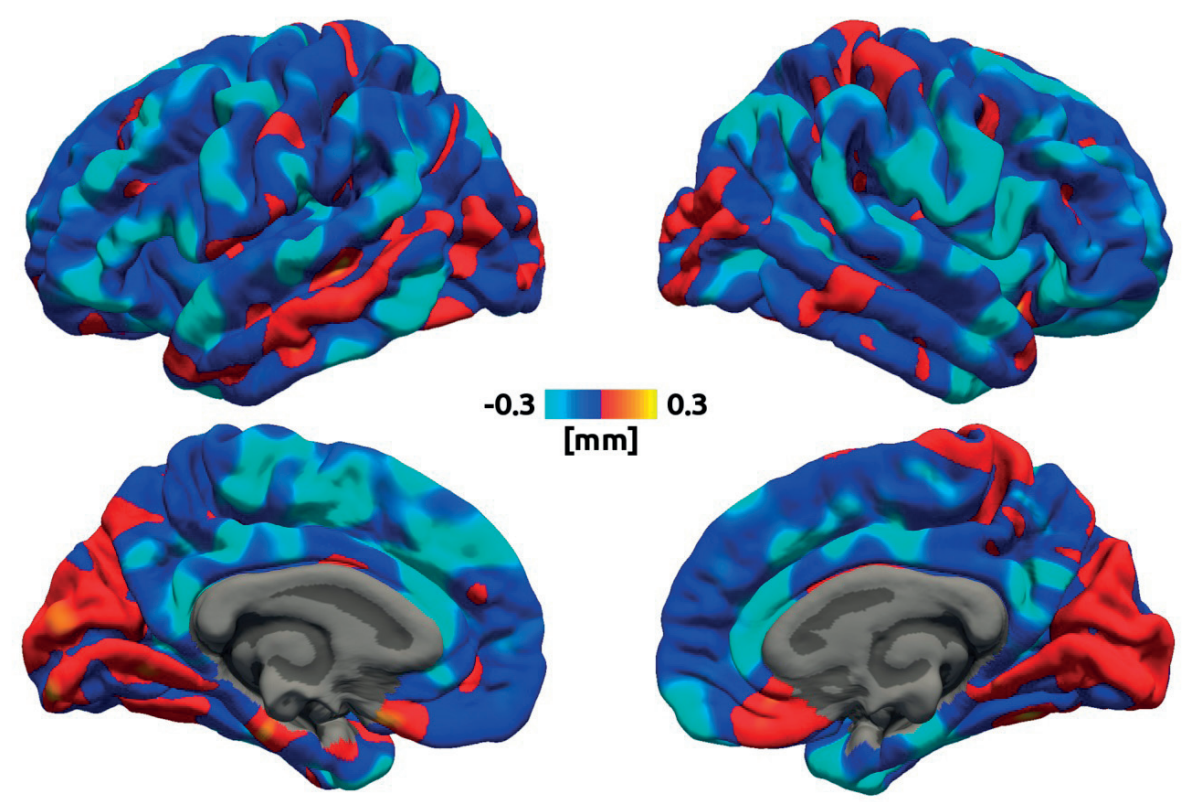

Fig. 1. Average cortical thickness difference between FOG- and FOG+. Colour overlay shows vertex-wise average difference in cortical thickness (in $\mathrm{mm}$ ) on top of an average three-dimensional cortical surface reconstruction. The cold colours represent greater atrophy in $\mathrm{FOG}+$ group, whereas warm colours represent greater atrophy in FOG- group. Left columns show the left hemisphere, right columns show the right one. 


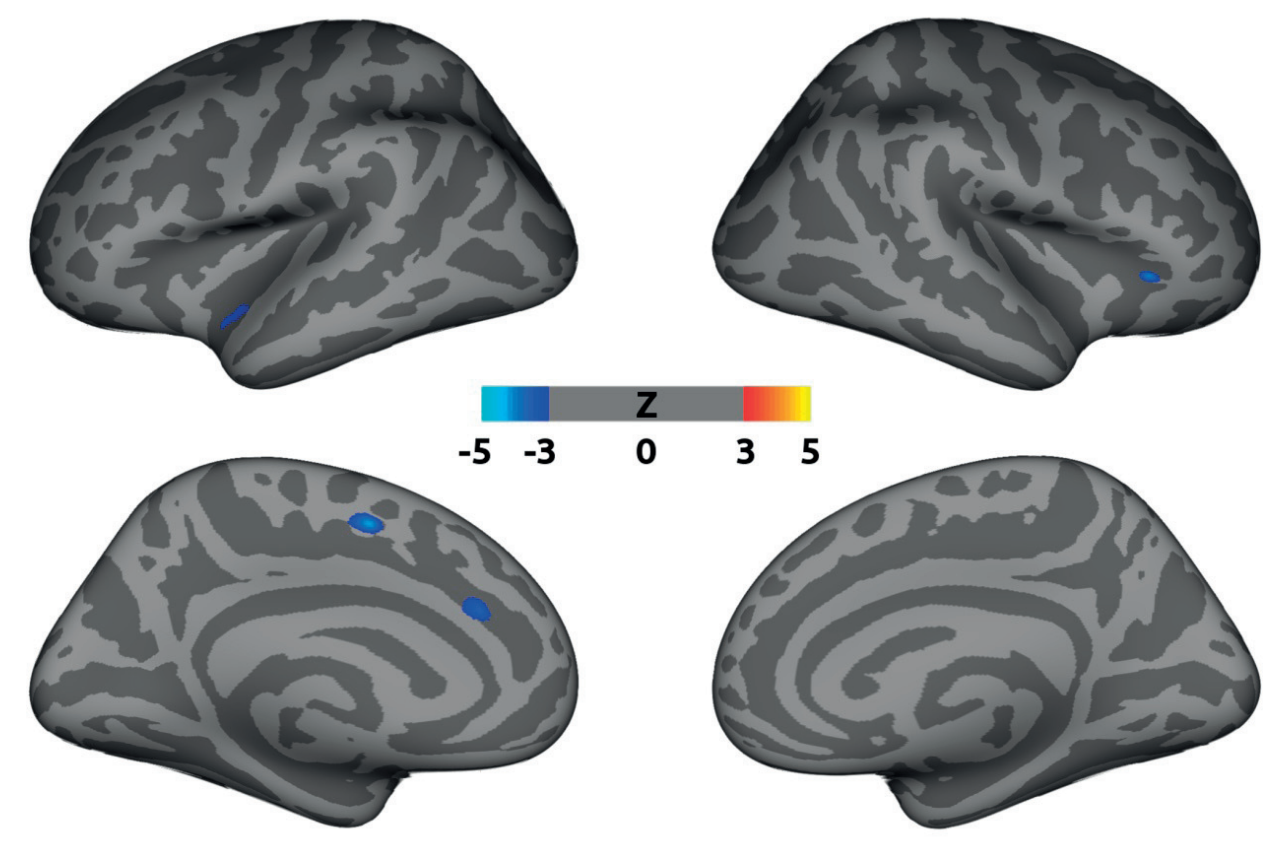

Fig. 2. Significant vertex-wise differences in cortical thickness FOG and FOG+. Colour overlay shows vertices of significant average difference in cortical thickness (represented by $\mathrm{Z}$ score, thresholded at $\mathrm{P}<$ 0.001, uncorrected) on top of an average inflated three-dimensional cortical surface reconstruction. The cold colours represent greater atrophy in $\mathrm{FOG}^{+}$group, whereas warm colours represent greater atrophy in FOG- group. Left columns show the left hemisphere, right columns show the right one.

labels not only confirmed significant differences in the left mid-anterior cingulate cortex, but also revealed significant cortical reduction in more posterior portions of the cingulate cortex bilaterally (Table 3 ). Again, no significant atrophy was detected in the FOG- group.

\section{DISCUSSION}

Pioneering VBM study combined with functional magnetic resonance imaging (fMRI) (ref. ${ }^{5}$ ) did not reveal any regional cortical atrophy specific for FOG on the whole-brain level, but described local GM atrophy in a small portion of the mesencephalic locomotor region (MLR) along with functional hyperactivation during walking imagery ${ }^{5}$. In contrast, when Tessitore et al. ${ }^{6}$ directly contrasted PD patients with and without FOG, they found GM atrophy in the left cuneus, lingual gyrus, precuneus, and posterior cingulate cortex. On the other hand, when comparing GM atrophy in PD patients with FOG to both control subjects and PD patients with no FOG, Kostic et al. ${ }^{7}$ observed atrophy of the left inferior frontal gyrus, left precentral gyrus, and left inferior parietal gyrus. Lastly, Herman et al. ${ }^{29}$ reported that FOG severity correlated with atrophy in the bilateral inferior parietal lobule, right angular gyrus, and bilateral caudate nuclei, although no significant group-wise differences were found. In this study, we used the FreeSurfer implementation of surfacebased analysis and our results demonstrate that patients with FOG exhibited atrophy of the bilateral cingulate cortex (mostly in mid-anterior part), left supplementary motor area (SMA), and right frontal operculum. In sum- mary, earlier publications reported rather complex pattern of functional and morphological changes ${ }^{6-11,29}$, suggesting a complex pathophysiological substrate of FOG. Whereas the automatic control of gait appears to be subserved by brainstem nuclei and their corresponding circuits, involvement of cortical brain structures is presumed in FOG because of frequent manifestation of freezing in situations with emotional or cognitive context.

Of these, emotional context seems to be a prominent factor for worsening of FOG. It was found that the FOG group reported significantly higher levels of anxiety compared to non-freezers in Martens et al. ${ }^{30}$. Additionally, over 230 freezing of gait episodes were elicited (in a sample of only 14 patients with FOG) when walking in the anxious environment. The study provided strong evidence that anxiety is an important mechanism underlying freezing of gait and suggested that increasing limbic load leads to increased freezing of gait and step-to-step variability. Indeed, parkinsonian pathology is responsible for the reduction in amygdalar volume and the concomitant development of anxiety symptoms ${ }^{31}$.

The cingulate cortex, a region of prominent atrophy in our study (blue in Fig. 1 and 2, Table 3 and 4), is an integral part of the limbic system, which is involved in emotion processing, learning and memory ${ }^{32}$. As such, the cingulate cortex receives inputs from the thalamus and the neocortex and projects to the entorhinal cortex via the cingulum. The anterior cingulate cortex is also responsible for changes in behaviour in response to unexpected outcomes, and it is therefore implicated in the so called salience network ${ }^{33}$. FMRI evidence also implicates the anterior cingulate cortex in modulating reward signals in the 
ventral striatum, effectively deducting the cost of cognitive control. Frank et al. ${ }^{34}$ have proposed that projections from dorsal anterior cingulate cortex to subthalamic nucleus (STN) specify the threshold for evidence accumulation before initiating a motor or cognitive response, and the efferents from STN implement this threshold. Functional imaging studies have suggested that the supracallosal anterior cingulate cortex and dorsolateral prefrontal cortex participate in tasks that require cognitive control. Cognitive control generally refers to a resource-limited system that guides complex voluntary actions and modifies habitual responses ${ }^{35}$. Lesions of the anterior cingulate and adjacent cortical regions cause apathy, amotivation, akinetic mutism that are problematic disease symptoms in many Parkinson's patients ${ }^{36}$. The mid-anterior cingulate has been already shown to be compromised functionally and/or morphologically in previous fMRI and VBM studies in FOG (ref. ${ }^{5,7}$ ). An impairment of its ample functions (emotional loops, reward system, salience network, and cognitive control) may thus consequentially lead to symptoms similar to FOG.

Other atrophic areas were found in the frontal lobe, which is the largest lobe in the human brain, yet it is often not specifically evaluated in routine neurologic examinations $^{37}$. Traditional classification systems divide the frontal lobes into the precentral cortex, orbitofrontal cortex, superior mesial regions and prefrontal cortex including the frontal operculum. Of these, the frontal operculum is a key node in a network for exerting control over cognitive processes and regulates increases and decreases of activity in multiple occipitotemporal cortical areas when task performance depends on directing attention to different classes of stimuli held in memory ${ }^{38}$. An atrophy in that area (Fig. 2) might be suggestive that an abnormal processing of those stimuli (i.e., obstacles, thresholds, etc.) might occur in FOG. Similar result of atrophy in the frontal operculum was reported by Herman et al. ${ }^{29}$.

A prominent regional atrophy was found at the superior frontal gyrus in the anterior supplementary motor area (SMA), shown in blue in Fig. 1 and 2. The SMA is crucial for planning and control of complex motor sequences. Previous studies showed that structural and functional abnormalities in this brain region are related to FOG in PD (ref., ${ }^{5,11}$ ). The SMA contributes to the anticipatory postural adjustment for step initiation, which is impaired in PD patients ${ }^{39}$. In the higher primates and in particular those with bipedal gait, SMA/PM (premotor area) projections to the pedunculopontine nucleus (PPN), in addition to the pontomedullary reticular formation, may play a crucial role of initiation of locomotion with appropriate postural control.

Although our analysis only revealed several regions of significant brain atrophy in FOG, the vast areas of statistically in significant decrease in cortical thickness (Fig. 1) are suggestive that our results are just a tip of an iceberg. Based on our results and the literature, we can suggest that cortical alterations not only in the SMA, but in a widespread network including frontal and parietal brain regions are involved in the pathophysiology of PDrelated gait impairment and FOG $\left(\right.$ ref. $\left.^{29}\right)$. For instance, perceptual malfunctions related to tissue loss in the inferior parietal lobe (IPL) contribute to FOG (ref. ${ }^{40}$ ). The IPL is activated during action execution and is involved in the sensory integration of perceptual spatiotemporal information. IPL atrophy may predispose patients to disrupted control of gait that leads to FOG. Specifically, selective attention allocation and maintenance, action processing and response inhibition, salient event encoding in the environment, and discrimination of left from right are regulated by the IPL and angular gyrus ${ }^{40}$. Although in general, the bilateral IPL has shown lower cortical thickness in FOG+ than in FOG- (Fig. 1), we did not confirm significant changes in that area.

The discrepancies between this and previous VBM studies might be well explained by methodological differences, since even minor changes in the analysis pipeline may significantly affect the VBM outcome ${ }^{4}$. Future studies, perhaps on bigger patient groups are needed to establish the ground truth to these results. However, the fully automated procedure implemented in FreeSurfer provides a rather consistent framework for future comparisons.

Although FOG remains a mysterious phenomenon, animal and imaging studies have started to unravel the mystery ${ }^{41}$. The outcome of these studies points to a dynamic process of hypo- and hyperactivation in cortical areas such as the SMA, anterior cingulate cortex, as well as subcortical areas such as the striatum and the mesencephalic locomotor region (MRL) including PPN. Nevertheless, the specific role of the PPN remains unclear. Basic science studies suggest the PPN is most involved in tone and balance more than in stepping ${ }^{38}$. Consequently, patients' studies hitherto suggest PPN stimulation may improve falls, more than FOG (ref. ${ }^{42}$ ). Control of locomotion is very complex, it is a network of multiple interacting brain areas using multiple neurotransmitters. Whether different phenomenological subtypes of freezing (akinetic, trembling, responsiveness to environment) have truly different pathologies, or whether they reflect different defective nodes of the same network, remains to be determined. Giving attention to other possibly relevant areas in the dynamic pathophysiological process of FOG, such as the SMA or the anterior cingulate cortex, may provide further direction in the development of effective therapies.

\section{Limitations}

MRI methods are likely the most currently used tool in neuroscience research. Despite progress in functional MR imaging over the past 20 years, there are still technical limitations that arise from the method itself. One limitation of this study is the small sample size, however it is comparable to previous studies ${ }^{6}$. Moreover, the optimized normalization procedures implemented in FreeSurfer improve the chance for group overlap of identical anatomical regions ${ }^{27}$.

\section{CONCLUSION}

The current study confirms that FOG is related, at least in part, to disruptions in the "executive-attention" 
network as shown by the regional cortical thickness changes in comparison to IPD patients without FOG. The most affected structures were the anterior and midanterior cingulate, and superior frontal gyrus (SMA). The grey matter damage is suggested to play a causative role in maladaptive neural compensation that may present transiently in the presence of acute conflicting motor, cognitive or emotional stimulus processing, thus causing acute network overload and resulting in episodic impairment of stepping. The responsible pathophysiological substrate of FOG is likely rather complex, it is not clearly understood, and further studies are important to investigate this mysterious phenomenon.

\section{ABBREVIATIONS}

BA, Brodmann area; FA, Flip angle; FLAIR, Fluid attenuated inversion recovery; FOG, Freezing of gait; FOV, Field of view; fMRI, Functional magnetic resonance imaging; GLM, General linear model; GM, Grey matter; IPL, Inferior parietal lobule; MMSE, Mini-Mental-State-Exam; MPRAGE, Magnetization prepared rapid acquisition gradient echo; MRI, Magnetic resonance imaging; MRL, Mesencephalic locomotor region; PD, Parkinson's disease; PM, Premotor area; PPN, Pedunculopontine nucleus; ROI, Region of interest; SD, Standard deviation; SMA, Supplementary motor area; STN, Subthalamic nucleus; TI, Inversion time; TE, Echo time; TR, Repetition time; VBM, Voxel-based morphometry.

Acknowledgement: This study was supported by grants of the Czech Health Research Council (AZV ČR) 1630210A and NV17-29452A. This work was also supported by the Institutional Support of the Research Organisation - Ministry of Health, Czech Republic, RVO - FNOL 2017.

Author contributions: All authors contributed equally to the manuscript.

Conflict of interest statement: The authors state that there are no conflicts of interest regarding the publication of this article.

\section{REFERENCES}

1. Giladi N. Medical treatment of freezing of gait. Mov Disord 2008;23(2):482-8.

2. Bloem BR, Hausdorff JM, Visser JE, Giladi N. Falls and freezing of gait in Parkinson's disease: a review of two interconnected, episodic phenomena. Mov Disord 2004;19:871-84.

3. Hausdorff JM, Schaafsma JD, Balash Y, Bartels AL, Gurevich T, Giladi $\mathrm{N}$. Impaired regulation of stride variability in Parkinson's disease subjects with freezing of gait. Exp Brain Res 2003; 149(2):187-94.

4. Henley SM, Ridgway GR, Scahill RI, Klöppel S, Tabrizi SJ, Fox NC, Kassubek J. Pitfalls in the use of voxel-based morphometry as a biomarker: examples from Huntington disease. AJNR Am J Neuroradiol 2010;31(4):711-9.

5. Snijders AH, Leunissen I, Bakker M, Overeem S, Helmich RC, Bloem BR, Toni I. Gait-related cerebral alterations in patients with Parkinson's disease with freezing of gait. Brain 2011;134:59-72.

6. Tessitore A, Amboni M, Cirillo G. Regional gray matter atrophy in patients with Parkinson disease and freezing of gait. AJNR Am J Neuroradiol 2012;33:1804-9.

7. Kostic VS, Agosta F, Pievani M, Stefanova E, Jecmenica-Lukic M, Scarale A, Spica V, Filippi M. Pattern of brain tissue loss associated with freezing of gait in Parkinson disease. Neurology 2012;78(6):40916.

8. Canu E, Agosta F, Sarasso E, Volonte MA, Basaia S, Stojkovic T, Stefanova E, Comi G, Falini A, Kostic VS, Gatti R, Filippi M. Brain structural and functional connectivity in Parkinon's disease with freezing of gait. Hum Brain Mapp 2015;36(12):5064-78.

9. Jha M, Jhunjhunwala K, Sankara BB, Saini J, Kumar JK, Yadav R, Pal PK. Neuropsychological and imaging profile of patients with Parkinson's disease and freezing of gait. Parkinsonism RelatDisord 2015;21(10):1184-90.

10. Rubino A, Assogna F, Piras F, Di Battista ME, Imperiale F, Chiapponi C, Spalletta G, Meco G. Does a volume reduction of the parietal lobe contribute to freezing of gait in Parkinson's disease? Parkinsonism RelatDisord 2014;20(10):1101-3.

11. Bruger F, Abela E, Hägele-Link S, Bohlhalter S, Galovic M, Kägi G. Do executive dysfunction and freezing of gait in Parkinson's disease share the same neuroanatomical correlaltes? J Neurol Sci 2015;356(1-2)84-7.

12. Dale AM, Fischl B, Sereno MI. Cortical surface-based analysis. I: Segmentation and surface reconstruction. Neuroimage 1999;9:17994.

13. Fischl B, Sereno MI, Dale AM. Cortical surface-based analysis. II: Inflation, flattening, and a surface-based coordinate system. Neuroimage 1999;9:195-207.

14. Desikan RS. An automated labeling system for subdividing the human cerebral cortex on MRI scans into gyral based regions of interest. Neuroimage 2006;31(3):968-80.

15. Destrieux C, Fischl B, Dale A, Halgren E. Automatic parcellation of human cortical gyri and sulci using standard anatomical nomenclature. Neuroimage 2010;53(1):1-15.

16. Bischoff-Grethe A, Ozyurt IB, Busa E, Quinn BT, Fennema-Notestine C , Clark CP, Morris S, Bondi MW, Jernigan TL, Dále AM, Brown GG, Fischl B. A technique for the deidentification of structural brain MR images. Hum Brain Mapp 2007;892-903.

17. Gelb DJ, Oliver E, Gilman S. Diagnostic criteria for Parkinson disease. Arch Neurol 1999;56:33-9.

18. Folstein MF, Folstein SE, McHugh PR. "Mini-mental state". A practical method for garding the cognitive state of patients for the clinician. J Psychiatr Re 1975;12:189-98.

19. Fahn S, Elton R, members of the UPDRS Development Committee. Unified Parkinson's disease rating scale. In: S Fahn, CD Marsden, DB Calne, M Goldstein (Eds) Recent developments in Parkinson's disease. Florham Park, NJ 1987;53-163.

20. Giladi N, McDermott MP, Fahn S, Przedborski S, Jankovic J, Stern $M$, Tanner C. Freezing of gait in PD: prospective assessment in the DATATOP cohort. Neurology 2001b;56:1712-21.

21. Giladi N, Shabtai H, Simon ES, Biran S, Tal J, Korczyn AD. Construction of freezing of gait questionnaire for patients with Parkinsonism. Parkinsonism Relat Disord 2000;6:165-70.

22. Nieuwboer A, Rochester L, Herman T, Vandenberghe W, Emil GE, Thomaes T, Giladi N. Reliability of the new freezing of gait questionnaire: agreement between patients with Parkinson's disease and their careers. Gait Posture 2009;30(4):459-63.

23. Segonne F, Dale AM, Busa E, Glessner M, Salat D, Hahn HK, Fischl B. A hybrid approach to the skull stripping problem in MRI. Neuroimage 2004;22:1060-75.

24. Fischl B, Salat DH, Busa E, Dieterich AM, Haselgrove C, Van der Kouwe A, Killiany R, D Kennedy, Klaveness S, Montillo A, Makris N, Rosen B, Dale AM. Whole brain segmentation: automated labeling of neuroanatomical structures in the human brain. Neuron 2002;33:341-55.

25. Fischl B, Liu A, Dale AM. Automated manifold surgery: constructing geometrically accurate and topologically correct models of the human cerebral cortex. IEEE Trans Med Imaging 2001;20:70-80.

26. Fischl B, Sereno MI, Tootell RB, Dale AM. High resolution intersubject averaging and a coordinate system for the cortical surface. Hum Brain Mapp 1999b;8:272-84.

27. Fischl B, van der Kouwe A, Destrieux C, Halgren E, Segonne F, Salat DH, Busa E, Seidman LJ, Goldstein J, Kennedy D, Caviness V, Makris $\mathrm{N}$, Rosen B, Dale AM. Automatically parcellating the human cerebral cortex. Cereb Cortex 2004b;1411-22. 
28. Fischl B, Rajendran N, Busa E, Augustinack J, Hinds O, Yeo BTT, Mohlberg H, Amunts K, Zilles K. Cortical folding patterns and predicting cytoarchitecture. Cereb Cortex 2008;18:1973-80.

29. Herman T, Rosenberg-Katz K, Jacob Y, Giladi N, Hausdorff JM. Gray Matter Atrophy and Freezing of Gait in Parkinson's Disease: Is the Evidence Black-on-White? Mov Disord 2014;29(1):134-9.

30. Martens KAE, Ellard CG, Almeid QJ. Does anxiety cause freezing of gait in Parkinson's disease? PLoS One 2014;9(9):e106561.

31. Vriend C, Boedhoe PS, Rutten S, Berendse HW, van der Werf YD, van den Heuve OAI. A smaller amygdala is associated with anxiety in Parkinson's disease: a combined Freesurfer-VBM study. J Neuro Neurosurg Psychiatry 2016;87(5):493-500.

32. Kozlovskiy S, Vartanov A, Pyasik M, Nikonova E, Velichkovsky B. Anatomical Characteristics of Cingulate Cortex and Neuropsychological Memory Tests Performance. Procedia - Social and Behavioral Sciences 2013;86:128-33.

33. Bonelli RM, Cummings JL. Frontal-subcortical circuity and behaviour Dialogues Clin Neurosci 2007;9(2):141-51.

34. Frank MJ. Computational models of motivated action selection in corticostriatal circuit. Curr Opin in Neurobiol 2011;21:381-6.

35. McDonald AW 3rd, Cohen JD, Stenger VA, Carter CS. Dissociating the role of the dorsolateral prefrontal and anterior cingulated cortex in cognitive control. Science 2000;288:1835-8.

36. Jordan LL, Zahodne LB, Okun MS, Bowers D. Hedonic and behavioural deficits associated with apathy in Parkinson's disease: potential treatment implications. Mov Disord 2013;28(9):1301-4.

37. Nelson SL Jr. Frontal lobe syndromes. Updated: Apr 11, 2016. Medscape. Available from: http://emedicine.medscape.com/ article/1135866-overview

38. Takakusaki K. Functional neuroimaging for posture and gait control. Mov Disord 2017:10(1):1-17.

39. Jacobs JV, Lou JS, Kraakevik JA, Horak FB. The supplementary motor area contributes to the timing of the anticipatory postural adjustment during step initiation in participants with and without Parkinson's disease. Neuroscience 2009;98:97-110.

40. Thaler A, Milerman A, Helmich RC. Neural correlates of executive functions in healthy G2019S LRRK2 mutation carries. Cortex 2013:49(9):2501-11.

41. Fasano A, Herman T, Tessitore A, Strafella AP, Bohne IN. Neuroimaging of freezing of gait. J Parkinsons Dis 2015;5(2):241-54

42. Snijders A, Takakusaki K, Debu B, Lozano A, Krishna V, Fasano A, Aziz T, Papa S, Factor S, Hallet M. Physiology of Freeing of Gait. ANN Neurol 2016;80:644-59. 